\title{
Polymer Packaging and Ejection in Viral Capsids: Shape Matters
}

\author{
I. Ali, ${ }^{1}$ D. Marenduzzo, ${ }^{2}$ and J. M. Yeomans ${ }^{3}$ \\ ${ }^{1}$ Department of Physics, College of Science, PO Box 36, Sultan Qaboos University, Al-Khodh 123, Oman \\ ${ }^{2}$ SUPA, School of Physics, University of Edinburgh, Mayfield Road, Edinburgh EH3 9JZ, United Kingdom \\ ${ }^{3}$ Rudolf Peierls Centre for Theoretical Physics, 1 Keble Road, Oxford OX1 3NP, United Kingdom
}

(Received 30 November 2005; published 26 May 2006)

\begin{abstract}
We use a mesoscale simulation approach to explore the impact of different capsid geometries on the packaging and ejection dynamics of polymers of different flexibility. We find that both packing and ejection times are faster for flexible polymers. For such polymers a sphere packs more quickly and ejects more slowly than an ellipsoid. For semiflexible polymers, however, the case relevant to DNA, a sphere both packs and ejects more easily. We interpret our results by considering both the thermodynamics and the relaxational dynamics of the polymers. The predictions could be tested with biomimetic experiments with synthetic polymers inside artificial vesicles. Our results suggest that phages may have evolved to be roughly spherical in shape to optimize the speed of genome ejection, which is the first stage in infection.
\end{abstract}

DOI: $10.1103 /$ PhysRevLett.96.208102

PACS numbers: 87.15.- v, 82.35.Lr, 87.15.Aa

In this Letter we study the packaging and ejection of polymers of different flexibility into and from spherical and ellipsoidal capsid shells. This is a model system for bacteriophages which consist of a semiflexible polymer DNA (the genome) packaged into a rigid container (the phage capsid) [1]. This system has recently attracted considerable theoretical attention [2-12]. Here we use a numerical approach which was developed in [13] where it was shown to reproduce the pauses during packing which have been observed experimentally [14]. Our main focus in the present work is on DNA ejection and on the impact of different capsid geometries on DNA packing and release.

The packaged DNA is subject to strong energetic and entropic penalties because it is contained within a capsid whose dimensions are typically smaller than the DNA persistence length $\sim 50 \mathrm{~nm}[14,15]$. This builds up an enormous internal pressure $\sim$ tens of atmospheres which the viruses or bacteriophages exploit to provide the simplest of attack strategies. Typically bacteriophages land on the surface of a bacterium and eject their genome into the host cytoplasm simply by taking advantage of the internal pressure which pushes the DNA out of the phage once the capsid is opened.

The diversity in the naturally occurring shapes of viral capsids is remarkable $[16,17]$. The shells of the phage DNA which infects prokaryots, like E. coli, B. subtilis, are spherical or quasispherical, stiff shells. For example, in the $\phi 29$ phage, the DNA is fed into a 54 by $42 \mathrm{~nm}$ icosahedral capsid [18]. On the other hand, viruses infecting higher eukaryots, which rely on a more complicated infection strategy than simple pressure-driven ejection, often have strikingly different, much more elongated shapes. An example here is the influenza virus which may be, e.g., $\sim 250 \mathrm{~nm}$ long and $\sim 100 \mathrm{~nm}$ wide [19]. Moreover, data on the internal volume of capsids, although sketchy, suggest that common spherical phages like T7, $\lambda$, and HK97 pack their genome at a density which is $\sim 10 \%-20 \%$ larger than that encountered for slightly aspherical phages like $\phi 29$ or T4 [see Table 2 in Ref. [2]]. Our results suggest a possible explanation for these observations.

Work in this area is particularly timely because in vitro single molecule experiments have led to significant quantitative insights on the dynamics of packaging and ejection in vivo. Smith et al. have measured the rate of packaging and the force of the motor for the $\phi 29$ bacteriophage [14], while more recent experiments have characterized DNA ejection from the T5 and $\lambda$ capsids $[20,21]$. Both the ejection and packaging rates have been shown to vary consistently and reproducibly during the various stages of these processes.

On the theoretical side, the effects of genome stiffness, excluded volume, and electrostatics on the DNA packaging process have been investigated by thermodynamic theories and simulations [2-8]. DNA ejection has also recently attracted a lot of attention among theorists: in particular, the roles of the buffer in DNA ejection experiments in vitro [9], the relation of ejection to translocation [10], and ratchet [11] models have been the subject of recent studies. Earlier work [12] pointed out that quasistatic analytic theories for DNA release require an assumption for the underlying main mechanism leading to friction during ejection.

In this Letter we use the stochastic rotation dynamics simulation model [22] to compare the way in which flexible and semiflexible polymers are packed into, and ejected from, spherical and ellipsoidal capsids of the same internal volume. Novel to this work are the explicit simulations of the ejection kinetics, which correctly capture nonequilibrium effects, and the focus on the impact of capsid geometry on the physics of DNA packing and releasing. We find that the slower relaxation times of the semiflexible chains leads to slower packing and ejection rates. A flexible polymer is ejected more quickly from an ellipsoidal shell 
than a spherical one. However, at first sight surprisingly, this situation is reversed for a semiflexible chain, which is ejected more quickly from a spherical shell. We argue that this is a consequence of balances between the thermodynamic force driving ejection and the ease with which the polymer can come to equilibrium within the confined space in the capsid. Recent advances in single molecule micromanipulation techniques [14] and in DNA ejection imaging and analysis $[20,21]$ put an experimental verification of these predictions within reach.

The polymer is a coarse-grained chain of $N=100$ beads joined by FENE springs, interacting via a potential $V$,

$$
4 \epsilon \sum_{i}\left(\sigma /\left|\vec{r}_{i}-\vec{r}_{i-1}\right|\right)^{12}+\kappa \Sigma_{i}\left(\vec{r}_{i+1}-\vec{r}_{i}\right) \cdot\left(\vec{r}_{i}-\vec{r}_{i-1}\right),
$$

where $\vec{r}_{i}$ is the position of the $i$ th bead. The first term is the repulsive part of a Lennard-Jones potential which generates excluded volume interactions between the beads. This is in tune with the experiments where repulsive interactions dominate. The potential parameters used were $\epsilon=k_{B} T$ and $\sigma=2.5 \mathrm{~nm}$. $\kappa$ in the second term in Eq. (1) is a bending rigidity which sets the persistence length $l \sim$ $\kappa \sigma / k_{B} T$. Here we set $l=0$ for a flexible polymer and $l=$ $10 \sigma$ for a semiflexible polymer. [We use $10 \sigma$ to compromise between reaching typical genomic stiffness $-20 \sigma$ under physiological conditions [14] — and feasible length and time scales in the simulations.] The updating of the beads' positions and velocities is performed using the velocity-Verlet molecular dynamics algorithm.

The capsid shapes, illustrated in Fig. 1, are described by

$$
f \equiv 1-\left[(x / a)^{2}+(y / b)^{2}+(z / c)^{2}\right]=0 .
$$

We choose $a=b=c=3.02 \sigma$ to model a sphere and $a=$ $b=2.6 \sigma$ and $c=4.07 \sigma$ for an ellipsoid. Each capsid is modeled as a hard shell with a hole that permits the entrance of one bead at a time. A repulsive force

(A)

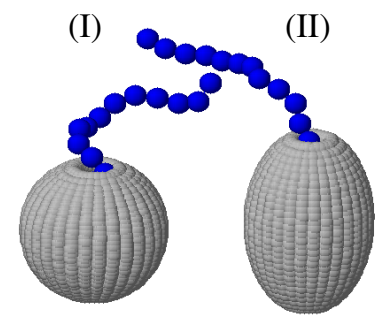

(B)
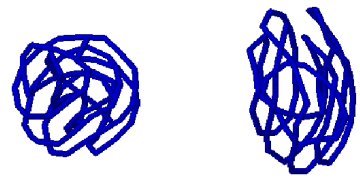

FIG. 1 (color online). Schematic representation of the simulation. A semiflexible polymer is first packed into and then ejected from a rigid capsid. The first row (A) shows the capsid and the dangling tail of the polymer close to the end of a packing run. The second row (B) shows the configuration of the polymer chain inside the capsid. Column (I) refers to a spherical capsid, and column (II) to an ellipsoidal one.
$k_{B} T /\left(\sigma f^{4}\right)$ is applied to any bead which is at a point for which $|f| \leq f_{0}$, where $f_{0}=0.2$ is a threshold. Our choices of $a, b, c$, and of $f_{0}$ lead to the same volume available to the chain for both shapes, which corresponds to a packing fraction of 0.4, comparable with previous numerical work and typical phage densities [3,4]. Qualitatively similar results to the ones reported below have been found with $N=80$ and $N=120$ with these capsid geometries, and with $N=200$ and a packing fraction of 0.4 .

The motor that feeds the polymer into the capsid is, in reality, extremely complex [15]. Here we use a simple model aimed at capturing the basic physics. Essentially the motor has to (1) capture a bead and (2) feed it into the capsid. This is accomplished by requiring the motor to apply a radial force (of magnitude $5 k_{B} T / \sigma$ ) if the bead enters a cylinder of radius $0.7 \sigma$ and length $\sigma$ with origin at the capsid entrance. The details of this mechanism do not affect our results. Once captured, the bead is packed by applying a constant force towards the center of the capsid. Our simulations allow us to identify the minimum motor force which is needed to achieve full packing. To estimate this, we ran a set of packing simulations at different motor force, and picked the lowest value of the force which still, on average, packed the whole chain. Flexible and semiflexible polymers are, respectively, found to require a minimum motor force of 16 and $20 k_{B} T / \sigma$ to be packed into a sphere, and of 18 and $26 k_{B} T / \sigma$ to be packed into an ellipsoid. In general, the difference between the forces corresponding to the sphere and the ellipsoid increases with packing fraction.

The polymer is coupled to a coarse-grained solvent model, stochastic rotation dynamics. This acts as a hydrodynamic thermostat allowing momentum transfer between beads and allowing flows to be set up in the surrounding fluid as a consequence of the bead motion. The solvent has a viscosity $\sim 5 \mathrm{cP}$, comparable to that of cytosol. The capsid is permeable to the solvent, which is the physical situation for phage capsids. (We measure force and time in simulation units in Figs. 2-4. One time and force simulation unit corresponds to $3 \mathrm{~ns}$ and $1.64 \mathrm{pN}$, respectively.)

The polymer is initially configured randomly except for the requirement that the first bead lies within the capsid and the rest outside. The polymer is then equilibrated in this position before opening the bead entrance and applying the feeding force. A single bead is left out to initiate ejection once the motor force is set to zero. This is done after leaving time for the polymer to equilibrate within the capsid.

Our simulations allow us to compare packing and ejection, a flexible and semiflexible polymer, and a spherical and ellipsoidal capsid. Figure 1 illustrates typical packed configurations for the semiflexible polymer-the polymers are ordered in spool-like domains (although not in an ideal inverse spool) as predicted theoretically $[3,6]$.

Figure 2 shows the number of packed beads as a function of time for both packing and ejection for the different chain flexibilities and capsid shapes. The motor force during 

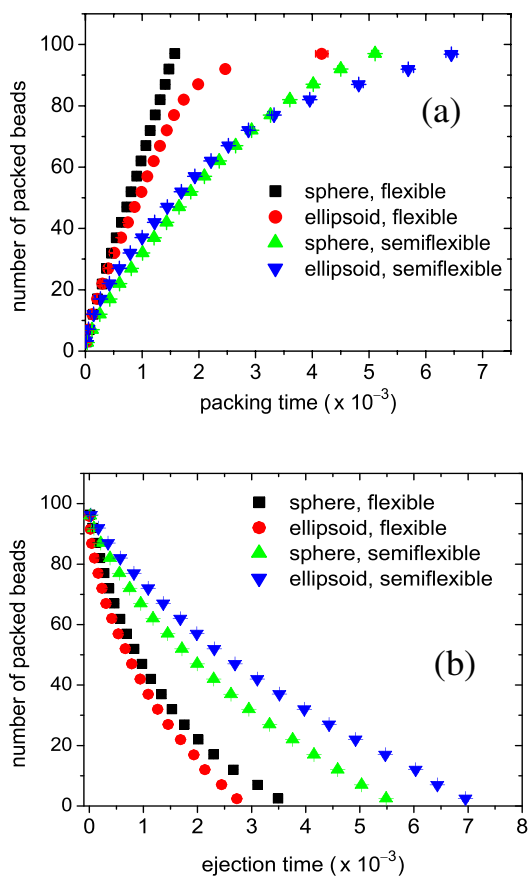

FIG. 2 (color online). Number of packed beads vs time during (a) packaging and (b) ejection for flexible and semiflexible polymers comparing a spherical and an ellipsoidal capsid.

packing was $26 k_{B} T / \sigma$-the minimum force to pack the semiflexible polymer into the ellipsoid, the case for which packing is hardest. The most immediately striking feature is that packing and ejection times are considerably faster for flexible polymers. This is because relaxation times increase as the polymer becomes stiffer and, e.g., once one bead has escaped it takes longer for the semiflexible
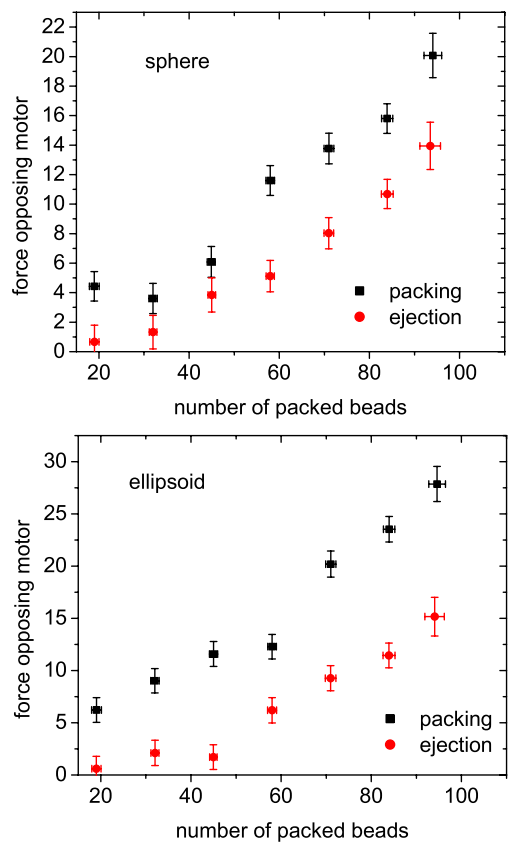

FIG. 3 (color online). Force opposing the motor (in units of $k_{B} T / \sigma$ ) during packaging and ejection for a semiflexible polymer in (top) a spherical and (bottom) an ellipsoidal capsid. chain to readjust itself so that a subsequent bead is in a position to escape.

Perhaps more surprisingly for flexible polymers the sphere ejects more slowly than the ellipsoid whereas for semiflexible polymers the reverse is true. The packing, on the other hand, proceeds more easily (it requires a smaller minimum force) and more quickly (at equal packaging force it takes less time) into the sphere whatever the flexibility of the polymer.

These results can be explained by considering a combination of entropic and dynamic arguments. Both flexible and semiflexible polymers lose more entropy when they are packed in an ellipsoid than when they are packed in a sphere. Therefore, based on entropic arguments alone, we would expect the polymers to pack more easily in a sphere and to be ejected more easily from an ellipsoid. This is the case for the flexible polymers [23].

For the semiflexible polymers, however, the sphere is faster both in packing and in ejection. We believe this to be primarily a consequence of two effects. First, the beads of the semiflexible polymer suffer more from the constraint of being in the ellipsoid when they try to rearrange themselves as beads are ejected. Second, the bending energy lost in packing is larger for the case of a sphere. These effects win over the entropic arguments and, for the semiflexible polymer, there is quite a pronounced advantage in ejection time for the sphere [24]. The difference in ejection times between the sphere and the ellipsoid increases with the aspect ratio of the ellipsoid.

To investigate these nonequilibrium effects further, we plot, in Fig. 3, the force opposing the motor during the
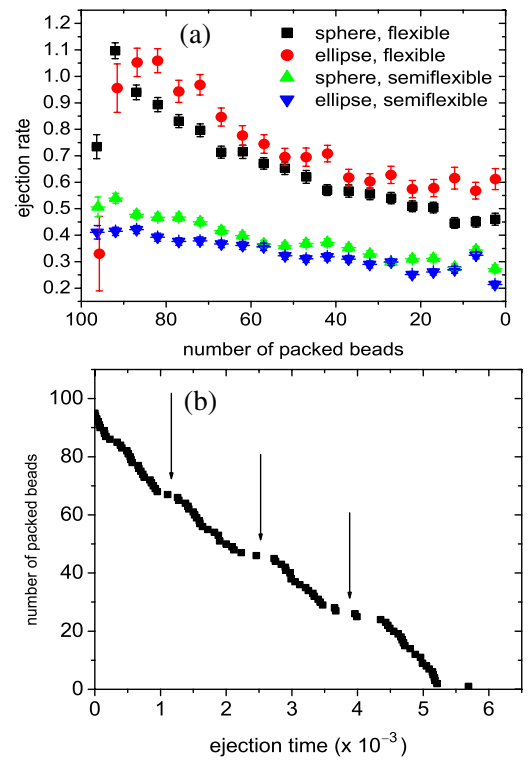

FIG. 4 (color online). (a) Ejection rate as a function of the number of packed beads for flexible and semiflexible polymers comparing a spherical and an ellipsoidal capsid. (b) Data from one individual simulation of the ejection of a semiflexible polymer from a sphere. Arrows indicate pauses (corresponding to rearrangements of the polymer inside the capsid). 
packaging and ejection of a semiflexible polymer for a sphere and an ellipsoid. We define such a force as the one felt by the bead inside the capsid which is closest to the motor at a given time. It includes the force due to local bending at the capsid entrance, the elastic force due to the springs acting on the bead under observation, and the overall Lennard-Jones repulsion of the other beads, both in the capsid and in the tail outside (i.e., we do not include the capsid contribution).

For both the capsid shapes there is hysteresis; i.e., the force during the packing is larger than the one felt during ejection. This shows that a significant portion of the resistance the motor has to overcome during packing is due to dynamic dissipative effects. The hysteresis is larger for the ellipsoidal capsid, supporting the assertion that chain rearrangements are more difficult in this case presumably due to the narrower space close to the capsid tip. That dynamic effects are important in our simulations can also be appreciated by noting that a set of simulations considering ejection from an ellipsoidal capsid with a hole on the long side yielded an ejection time comparable to that found from the sphere.

Typically, experiments report packing rates as a function of the number of packed beads [14] and we therefore present similar data for ejection in Fig. 4(a). The ejection rate decreases as the number of packed beads decreases for the flexible chain. This is because the force driving the ejection is the entropic penalty of confinement which decreases with decreasing packing fraction. For the semiflexible chain, the rate decreases appreciably less quickly suggesting that entropic considerations are less dominant. One can speculate that the decreasing entropic force is offset by easier rearrangements within the capsid as it empties.

The data in Figs. 2(b) and 4(a) are averaged over many runs and thus the curves appear continuous. However, individual runs indicate that, just as for packing, there are pauses in ejection as the polymer rearranges itself within the capsid [Fig. 4(b)].

In summary, we have compared the packing and ejection of flexible and semiflexible polymers within phage capsids of variable geometry. The behavior is influenced not only by thermodynamic considerations but also by the relaxation time of the polymers as they try to rearrange themselves within the confined capsid geometry. For semiflexible polymers we find that a spherical shape leads to fastest ejection. One might speculate that this is one of the reasons why most phages have evolved to be roughly spherical in shape. We note, however, that other phages, not relying on pressure to eject their genome [25], are still spherical, thus suggesting that the mechanics and energetics of the proteins making up a viral capsid are equally important in ultimately determining shape. We also found that nonequilibrium effects hamper packing into an ellipsoidal phage, which may explain why aspherical capsids contain less DNA. Our simulations suggest a series of single molecule biomimetic experiments in which the dy- namics of polymers of variable flexibility undergoing packing-ejection cycles into and out of vescicles of controlled shape are studied.

This work was supported by EPSRC Grant No. GR/ R83712/01 and by ONR. We thank R. Golestanian for useful discussions.

[1] B. Alberts et al., Molecular Biology of the Cell (Garland Science Publishing, New York, 2002), 4th ed.

[2] P. K. Purohit et al., Biophys. J. 88, 851 (2005).

[3] J. Kindt et al., Proc. Natl. Acad. Sci. U.S.A. 98, 13671 (2001); S. Tzlil et al., Biophys. J. 84, 1616 (2003).

[4] P. K. Purohit, J. Kondev, and R. Phillips, J. Mech. Phys. Solids 51, 2239 (2003); Proc. Natl. Acad. Sci. U.S.A. 100, 3173 (2003).

[5] T. Odijk, Phil. Trans. R. Soc. Lond. A 362, 1497 (2004).

[6] J. Arsuaga et al., Biophys. Chem. 101, 475 (2002).

[7] J. Arsuaga et al., Proc. Nat. Acad. Sci. U.S.A. 102, 9165 (2005).

[8] D. Marenduzzo and C. Micheletti, J. Mol. Biol. 330, 485 (2003).

[9] A. Evilevitch et al., Proc. Natl. Acad. Sci. U.S.A. 100, 9292 (2003).

[10] M. Muthukumar, Phys. Rev. Lett. 86, 3188 (2001).

[11] M. M. Inamdar, W. M. Gelbart, and R. Phillips, q-bio.BM/ 0507022.

[12] I. S. Gabashvili and A. Y. Grosberg, J. Biomol. Struct. Dyn. 9, 911 (1992).

[13] I. Ali, D. Marenduzzo, and J. M. Yeomans, J. Chem. Phys. 121, 8635 (2004).

[14] D. E. Smith et al., Nature (London) 413, 748 (2001).

[15] A. A. Simpson et al., Nature (London) 408, 745 (2000).

[16] T. S. Baker, N. H. Olson, and S. D. Fuller, Microbiol. Mol. Biol. Rev. 63, 862 (1999).

[17] R. Twarock, J. Theor. Biol. 226, 477 (2004).

[18] Y.Z. Tao et al., Cell 95, 431 (1998).

[19] Universal Virus Database, http://www.ictvdb.rothamsted. ac.uk/index.htm.

[20] S. Mangenot et al., Curr. Biol. 15, 430 (2005).

[21] M. de Frutos, L. Letellier, and E. Raspaud, Biophys. J. 88, 1364 (2005).

[22] A. Malevanets and R. Kapral, J. Chem. Phys. 110, 8605 (1999); N. Kikuchi et al., J. Chem. Phys. 119, 6388 (2003); T. Ihle and D.M. Kroll, Phys. Rev. E 63, 020201(R) (2001).

[23] This is in line with results on the 2D lattice; see A. Jaeckel and J. Dayantis, Macromol. Theory Simul. 10, 461 (2001).

[24] Note that the theory in [4] does predict a dependency of the packing force and ejection time on capsid shape. For example, the packing force would be predicted to increase - and the ejection time to shorten-if the capsid were a cylinder of increasing aspect ratio, due to the different bending felt by the genome in these cases. However, a quantitative comparison with our results is problematic as our model considers less stiff chains, includes nonequilibrium relaxation effects, and the ideal coaxial spool model is not assumed.

[25] I. J. Molineux et al., Mol. Microbiol. 40, 1 (2001). 